\title{
El sistema penal es un espejo de la injusticia social: las deudas con las mujeres en la cárcel
}

\section{The Penal System Is a Mirror of Social Injustice: The Debts with Women in Prison}

\author{
Claudia Palma Campos ${ }^{l}$ \\ Universidad de Costa Rica \\ Costa Rica
}

\begin{abstract}
Resumen
El presente artículo tiene como objetivo analizar el contexto donde ocurre el sistema penal, lo que moviliza la aplicación de ciertas normativas y sobre las nociones morales y culturales de su aplicación, así como la comisión de un delito, específicamente en relación con las mujeres en la cárcel. Se basa en la experiencia directa de la autora como antropóloga y feminista en los centros penales de Costa Rica, principalmente y en el trabajo con mujeres privadas de libertad por casi diez años. Este documento trata de mirar el lugar social que han ocupado las mujeres y cómo este se refleja cuando llegan a la cárcel, cómo pueden ser miradas y tratadas y cómo sus delitos, sus penas y sus reclamos son producto, de una u otra manera, de la desigualdad y la injusticia social reflejada en la injusticia penal. Se plantea una discusión sobre diferentes experiencias en el sistema penal en Costa Rica y se formulan serios problemas que refuerzan la desigualdad social, como el sexismo y carencia de enfoque de género. Se concluye que las mujeres que están en la cárcel no son diferentes a otras mujeres oprimidas socialmente en el país. Sus voces, sus necesidades y sus tristezas pertenecen al mismo orden de aquellas que día a día tratar de idear maneras para pertenecer, social, económica y políticamente
\end{abstract}

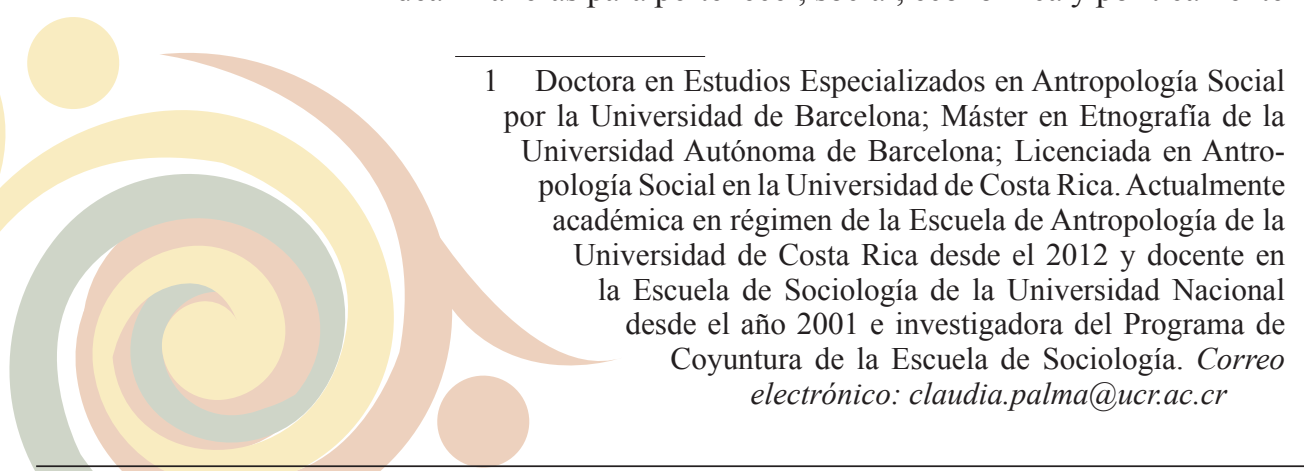


a un país que les ha negado oportunidades. Se plantea como una obligación retomar su voz para contribuir a la equidad social.

Palabras clave: feminismo; cárcel; mujeres; etnografía; criminología crítica; sistema penal.

\begin{abstract}
This article aims to analyze the context where the criminal system occurs, which enables the application of certain regulations. It also analyzes the moral and cultural notions of the implementation of these regulations, as well as the commission of a crime, specifically concerning women in jail. It is based on the author's direct experience as an anthropologist and feminist in Costa Rica's prisons, mainly working with women deprived of their liberty for almost ten years. This paper tries to look at the social place that women have occupied and how this is reflected when they arrive in prison, how they can be looked at and treated and how their crimes, their punishments, and their claims are the product, in one way or another, of inequality and social injustice reflected in criminal injustice. There is a discussion about different experiences in the penal system in Costa Rica, and serious problems are formulated that reinforce social inequality, such as sexism and lack of a gender approach. In conclusion, women in prison are not different from other socially oppressed women in the country. Their voices, their needs, and their sorrows belong to the same order as those that, day by day, try to devise ways to belong, socially, economically and politically, to a country that has denied them opportunities. It is proposed as an obligation to retake their voices to contribute to social equity.
\end{abstract}

Keywords: feminism; prison; women; ethnography; critical criminology; penal system.

\title{
Introducción
}

Es importante situar, en este inicio, el lugar desde el cual me posiciono y, por lo tanto, desde el que escribo. Este es uno muy particular, como antropóloga y feminista. Ambos posicionamientos políticos implican una forma de ver la realidad cotidiana, a la vez que toman partido sobre ella. En el tema específico sobre la privación de libertad, ya sea de hombres o de mujeres, me atraviesa la experiencia de haber empezado a escuchar sus historias desde hace varios años y tratar de ubicarlas en un contexto social, político, económico y, claro, cultural. Es por esto que la perspectiva que me interesa sobre el sistema penal no es sobre el acatamiento a la norma, el impacto social del crimen o lo correcto/incorrecto de un delito, sino acerca del contexto en que ocurre, lo que moviliza la aplicación de ciertas 
normativas, según qué contextos, así como las nociones morales y culturales de su aplicación, al igual que la comisión de un delito. Este último está íntimamente relacionado con las experiencias de las personas, cómo lo viven, cómo le dan un concepto y un lugar en su mundo.

Por otro lado, vale también la pena aclarar que la antropología no juzga las historias, no hay unas más o menos importantes que otras. Su tarea es ubicarlas en el tiempo y el espacio; es decir, en el contexto político y cultural en el que son escuchadas, para comprender y transmitir algo más que la voz de quienes las dicen. Este documento lo que tratará es de mirar el lugar social que han ocupado las mujeres y cómo este se refleja en el sitio que ocupan cuando llegan a la cárcel, cómo pueden ser miradas y tratadas, cómo sus delitos, penas y reclamos son producto, de una u otra manera, de la desigualdad y la injusticia social manifestada en la injusticia penal. Estas reflexiones son secuela de la experiencia directa que he tenido en los centros penales de Costa Rica, principalmente, y del trabajo más intenso con mujeres privadas de libertad por casi diez años.

\section{El sistema penal es un espejo de la desigualdad social}

Quisiera empezar diciendo que existen muchas mujeres que desaparecen del mapa social, que dejan de existir en el momento en que llegan a la cárcel, producto de la desigualdad e inequidad estructural de la que proceden. Ya antes de la cárcel tampoco eran visibles, pero en ella se esfuman. La equidad social es un paso que va más allá de la igualdad de derechos, pues implica los esfuerzos necesarios para garantizar que exista menos margen en las condiciones sociales que producen desequilibrio y sufrimiento.

Hablar de equidad social significa hacer lo necesario para que las personas, en condiciones de disparidad económica, social, política y cultural, puedan tener acceso a todos los recursos sociales dispuestos por un país, con miras al bienestar. Lejos de ello, los recursos de la equidad se han enfocado en mantener a quienes son más iguales en su condición social, mientras que la inequidad se profundiza cuando se atraviesa por elementos como el sexo, la procedencia, la clase, el origen, el color y el género.

La idea se traslada al sistema penal en su ejercicio de la justicia. Este no administra sentencias, sino a personas con ellas, quienes tienen una historia de injusticia encima. Si bien la justicia tendría que ser el producto de la lectura correcta del ámbito social y cultural en el que ocurren los delitos, se convierte en un mecanismo de castigo que recoge las principales opresiones de la sociedad contemporánea, 
colmadas de las particularidades con que cada grupo social matiza ya no la pena, sino el encierro y la sanción. Y, si es por colocar las particularidades de la relación sexo-género, la opresión estructural de muchas mujeres se refleja en los delitos y, a la vez, en la forma como el sistema penal resuelve la ejecución de su pena, lo cual trataré de ejemplificar más adelante.

Primero es importante aclarar que, cuando se afirma que el sistema penal reproduce la desigualdad social, no es una frase sin sentido o un lugar común feminista sobre la desigualdad, sino que tiene una historia, con un origen sobre la idea de quiénes son las personas sujetas de derecho y de quiénes son iguales, a partir de la construcción del "sujeto político". Esta es una noción producto del andamiaje y de la herencia de nuestro Estado-nación, por lo tanto, consustancial a la normativa jurídica.

Este sujeto político no es cualquier persona, sino uno "civilizado", lejano a la barbarie. Para Norbert Elias (1993), en el proceso de asentamiento de la modernidad, es la idea de civilización la que termina de definir una moral y un comportamiento acorde con el tipo de sociedad naciente de la época; a saber: puritana y con un sujeto "civilizado", regido por la noción de distinción social. Puritanismo y modernismo son cara de la misma moneda, pues, en estas sociedades, producto de las cuales es el Estado moderno, tanto la razón como la fe moldearon las voluntades individuales, a través de complejos y cotidianos mecanismos de control, que luego conciben las instituciones necesarias para consolidarlos. Tanto la familia, como la iglesia y las leyes modificaron y regularon las conductas y los cuerpos, la sexualidad y el prestigio, así como la palabra y la verdad.

Fueron los "hombres de letras" quienes construyeron el Estado. Ellos eran los que tenían la palabra y, por lo tanto, la opinión y el poder: hacían política y justicia. Marcada esta brecha, del otro lado estaban las personas a las cuales gobernaban y sobre quienes tomaban decisiones: la prole, el pueblo, la muchedumbre, la barbarie, es decir, aquellas a las que se catalogaba como una masa iletrada o "incivilizada", personas definidas como seres inferiores, carentes y dependientes, no legítimos para formar Estado y tomar decisiones.

Este proceso es muy interesante en la historia, pues con el Estado se instaura el contrato y con él sus sujetos contractuales: las personas libres que, en ese caso, por defecto más que por derecho, eran solo los hombres. Tal como lo menciona Carole Pateman (1988) el hombre libre es aquel capaz de negociar, de instaurar el contrato que solo se puede firmar con otro sujeto libre. Las personas no libres, 
tutelables, no iguales ni merecedoras de derechos, quedaron afuera de la foto de la equidad.

La justicia se instaura entre iguales, tal como hoy, no entre los tutelables. Es Ana María Fernández (1993) quien habla del tutelaje como una institución jurídica, cuyo objetivo es la guarda de las personas o bienes que son "incapaces de gobernarse a sí mismos". Si un contrato necesita dos sujetos libres, iguales en tanto individuos, la tutela requiere un sujeto libre y otro incapacitado o inhabilitado para el ejercicio de la libertad. En esta incapacidad social, subjetiva y política, que impedía la participación en el espacio público, han estado, junto a las mujeres, los pobres, es decir, la prole y aquellos extraños que contravenían las normas sociales. Esta es solo una parte del sostén de la desigualdad de la población femenina, que luego se profundiza en las creencias que consolidan parámetros discriminatorios en nuestra sociedad, como el sexismo, o bien el racismo y el clasismo. Con esto no se afirma que los hombres estén siempre mejor, pero sobre las mujeres recae una condena moral, cuando se faltan a los valores de sujeción, sumisión, ideal de bondad o entrega, y todos en su conjunto se profundizan cuando ellas comenten un delito.

Son construcciones socioculturales y políticas que no únicamente afectan la vida cotidiana, sino también la toma de decisiones y visiones de mundo institucionales. Por ejemplo, pensando en la construcción de la criminalidad, Dolores Juliano (2004) nos recuerda que, a finales del siglo XIX, la prensa europea inventa las "clases peligrosas" que comprendían a vagabundos, jugadores, personas pobres $\mathrm{y}$, por supuesto, prostitutas. Estas personas eran criminalizadas por pertenecer a sectores que se denominaban "atrasados", pero no solo eso, eran diferenciados y excluidos por la imposición de un sistema económico productivo. Asimismo, contrariaban la norma de una moral implantada, una sujeción a la que algunos no respondían. En tanto cada sociedad tiene su sistema de sanciones, acorde con su estructura cultural, sus valores y sus creencias, esas personas recibieron algún tipo de castigo, pues no tenían los principios morales ni los económicos aparejados. Tanto el señalamiento como la estigmatización y, por lo tanto, la construcción de peligrosidad responden a cada sociedad. Si el delito es producto del poder, para decir de una moral específica que protege a los iguales, ¿quiénes le hacen daño a estos últimos? En otras ocasiones, he recurrido a un ejemplo, como consecuencia de la investigación del Programa de las Naciones Unidas para el Desarrollo Humano (PNUD) (2005), sobre la desigualdad en el país, en el que se determinó cuál es el sujeto convertido en una amenaza que provoca inseguridad 
y temor: fue el drogadicto aquel que acumuló el mayor porcentaje en cuanto a la posibilidad de cometer actos violentos o delictivos.

Este porcentaje permite comprender que tanto el delito como la penalización de hoy responden a dos fenómenos que se han profundizado en este inicio del siglo XXI: una creciente desigualdad social, que deviene de la violencia estructural, y el impacto tanto económico como político de la confrontación entre el norte y el sur, con uno de sus productos estrella, la (in)moral guerra contra las drogas en el nivel mundial. Es por la figura de miedo que desprende un drogadicto y, por lo tanto, su criminalización o estigmatización, que los porcentajes de delitos están asentados en estos dos valores económicos y morales: delitos contra la propiedad y delitos contra las drogas. En el país, ambos suman al menos el $62 \%$ del total cometido entre hombres y mujeres (Sistema Integral de Atención Penitenciaria (SIAP), 2019).

De esta manera, se mezclan, históricamente, varios elementos que permiten comprender los tipos de delitos cometidos por las mujeres: exclusión social, a partir de la invisibilidad de las mujeres como sujetas políticas, que las coloca, según su procedencia y sus oportunidades, en una fácil línea de cruzar hacia la marginalidad; una profunda estigmatización sexista sobre su subjetividad y sus actos, que las caracteriza como personas dependientes, sin voz, tutelables y, por lo tanto, manipulables; un contexto político y económico que profundiza la desigualdad y promueve el asentamiento de la economía de las drogas, en aquellos espacios marginalizados, donde el Estado, como garante de equidad, ha dejado de tener presencia.

\section{Explicaciones sexistas a delitos estructurales: las faltas sociales de las mujeres}

Dicho lo anterior, quisiera comentar sobre el impacto de que sean los delitos de tráfico de drogas aquellos por los que las mujeres llegan más a la cárcel en Costa Rica y en América Latina. Y es que, en el caso de ellas, están subrepresentadas en el sistema penal; no suman más del $6 \%$ de la población total en la cárcel; sin embargo, están sobrerrepresentadas en el delito del tráfico de drogas, en el que suman un $55 \%$, seguido del $25 \%$ en delitos contra la propiedad (SIAP, 2019). Las faltas por este último están tipificadas en la Ley 8204 "sobre estupefacientes, sustancias psicotrópicas, drogas de uso no autorizado, actividades conexas, legitimación de capitales y financiamiento al terrorismo" (Asamblea Legislativa, 2001). La gran mayoría de las mujeres se vincula a la venta de pequeñas cantidades de drogas o bien al ingreso de sustancias a un centro penal, ocultamiento, 
transporte, entre otros. Esto quiere decir que los recursos institucionales sobre la prisión están siendo dirigidos a la atención de un delito producto de la inequidad estructural. A la vez, la concentración de los discursos sobre la delincuencia tampoco puede obviar que es el tráfico de drogas el que tiene un peso en el análisis ofrecido cuando se habla de aquella en el país. Hacerlo significa descontextualizar las condiciones en que la trasgresión se produce.

Por ser un delito que está vinculado a un aparato económico y moral donde se asienta, y en vista de que es coherente con la cada vez más profunda desigualdad estructural, siempre me ha llamado poderosamente la atención la forma de calificar el acto cuando lo ejecutan las mujeres. La explicación tradicional se ha fundamentado en que ellas son obligadas por otras personas a cometerlo (Instituto Costarricense sobre Drogas (ICD), 2009; Torres, 2008). Es como si la propia condición de opresión de las mujeres y su contexto no fueran suficientes para determinar involucrarse en una falta penal, sino que lo prioritario radicaría en su sumisión y acato a lo que los otros les manden, mismo parámetro del tutelaje. Este marco de referencia hace pensar en la condición de ingenuidad con que han sido tachadas las mujeres, como sujetas sociales no suficientemente maduras, como para tomar una decisión de tal envergadura. Si esta es la base de un delito, es fácil pensar que pueden ser obligadas o manipuladas emocionalmente por, principalmente, algún hombre, pareja, hijo o familiar, para cometerlo.

Si para comprender la comisión del tráfico de drogas se reduce el análisis a lo simbólico del acato a la identidad sumisa de las mujeres, entonces se elimina el contexto sociocultural, de opresión social y económica, a una decisión personal de manipulación. En mi experiencia de trabajo de campo con las mujeres por tráfico de drogas, una buena parte de ellas, sino la mayoría, no menciona la manipulación, lo cual no quiere decir que no exista. Alguna habló de amenazas, por parte de una banda de narcotráfico de su barrio, pero no de una pareja. El resto de mujeres que he escuchado hace referencia a una decisión tomada en una compleja situación económica. Claro, si el tráfico no estuviera presente en su vida cotidiana, es difícil acceder a él. En este sentido, es una determinación de contexto, proveniente del lugar en el cual el tráfico se muestra como opción económica, no de vida (Palma, 2018). Las anteriores ideas, las he tratado de desarrollar con más profundidad en otros momentos, pero acá lo que me interesa comunicar es cómo poner atención al marco de referencia sobre el que se asienta la percepción sociocultural acerca de las acciones de las personas; en esta última, sin lugar a dudas, se asienta la corrección institucional sobre las mujeres. 
Otra idea impactante que recorre la vinculación de las mujeres al tráfico de drogas es que ellas lo hacen porque es un trabajo fácil y les deja dinero fácil (Palma, 2018). Este sea tal vez uno de los estereotipos con un fuerte sesgo de género en detrimento de las mujeres, de los más sexistas y violentos. La relación entre dinero fácil-mujer fácil se monta en todo el andamiaje que ubica a la población femenina como dependiente económica y emocional. Con ello, vuelve a ocultar el contexto en el cual se comete el delito, a la vez que se encubre la historia de trabajos precarios, sueldos miserables y sin garantías sociales de las que ellas provienen. Las mujeres, aun cuando cometen un delito, han trabajado mucho, antes de ingresar a la cárcel, a pesar de que ellas mismas no se reconozcan en la labor. Muchas veces solo dicen que son amas de casa, porque decir "soy ama de casa", en una sociedad que ve el cuido del hogar como un valor social femenino, es más digno que "vendo melcochas en la calle o soy prostituta".

El tráfico de drogas es uno más, y muy mal pagado; es el último eslabón económico de la economía de las drogas, pero tan explotador como todas las otras tareas informales, a las que han tenido acceso por su misma condición opresiva social y monetaria. Pero tampoco nos equivoquemos. Las mujeres no cometen delitos por ser pobres, sino por opresión, por violencia económica y estructural. Si las mujeres delinquieran solo por ser pobres, las cárceles estarían más llenas de ellas, tal y como lo apunta Dolores Juliano (2011), pues, además, son estructuralmente más pobres que los hombres; es decir, el impacto de la inopia es más fuerte en las mujeres. Sin embargo, estas, por pobres, no eligen delinquir, porque hacen otras mediciones para salir de su situación opresiva económica, tanto que recursos monetarios socialmente no estigmatizados, como la prostitución o la venta de pequeñas cantidades de drogas, son el último recurso y no el primero.

Existe otra situación en la que se conjuga el ejercicio explotador y simbólico que denigra a las mujeres vinculadas al tráfico de drogas: la cosificación genital de la dinámica de ingreso de drogas a los centros penales. La gran mayoría femenina que lleva drogas a la cárcel lo hace mediante su cuerpo, introduciendo en sus vaginas un cartucho de sustancias sellado con un condón. La dinámica de sacarlo y entregarlo es otro tema. Si ya con este hecho no se lee el aparato opresor de género, sexista y violento de las drogas, es muy difícil hacerlo con otro recurso. Si bien un tipo distinto de vinculaciones al tráfico, como la venta directa, al menudeo, se escapa del control institucional penal, sigo sin comprender cómo la introducción a la cárcel es un recurso tan asentado y útil. Con esto no pretendo que sea una invocación al control y la represión, sino una reflexión a la ganancia del sistema penal con el ingreso de drogas, que además se sostiene en la violencia contra las mujeres. En alguna ocasión, me atreví a decir que este recurso se 
convierte en una penetración pública, sin gozo y violenta, permitida y avalada por el sistema penal; solo disfrutada por la gran estructura de la economía del tráfico que usa, para su deshecho, los cuerpos de las mujeres.

\section{Penas y lamentaciones: la deuda del enfoque de género en el sistema penal}

A partir de estos aspectos, quisiera anotar una serie de puntos, más preguntas que respuestas, sobre lo que he observado en la dinámica carcelaria y en el trato a las mujeres, no como una forma de señalarlos para decir que están mal, sino como una manera de visibilizarlos desde un punto de vista antropológico, el cual no pertenece al sistema que juzga a las mujeres ni tampoco pertenece al medio que las encarcela. Incluso, lo que miro lo hago con un profundo respeto hacia las personas quienes ofrecen su atención a la privación de libertad, quienes cargan una responsabilidad muy grande, pero no por eso están exentas de formar parte del círculo de opresión institucional.

Tal vez habría que empezar diciendo que el sistema penal y de justicia sigue en deuda con las mujeres encarceladas, al no ofrecerles un espacio que, al menos, cuente con las condiciones mínimas y de atención específica para ellas. Al final del gobierno de Solís Rivera (2014-2018), se aprobó, con la instrucción de su Ministerio de Justicia, la construcción de una Unidad de Atención Integral (UAI) para mujeres, lo cual comportaba un gran avance en el camino a la equidad y al tratamiento penitenciario para las privadas de libertad.

Si bien una UAI no resuelve el problema de exclusión social previo, sí podía mejorar el conflicto de exclusión social futuro, al concentrarse en las condiciones particulares de los delitos de las mujeres que ya mencionamos, pero, también, en la construcción conjunta de oportunidades que les permitieran volver a la sociedad en mejores circunstancias y, ojalá, con mayores posibilidades. No obstante, la decisión de la administración Alvarado Quesada (2018-2022), en manos de su Ministra de Justicia Marcia González Aguiluz, decide traerse abajo la construcción de la UAI para las mujeres, por un ajuste presupuestario, según lo ha declaró en medios de comunicación (Cascante, 2018). Algo que se veía como un avance social y de género es reducido a un tema económico donde pareciera que las mujeres y sus condiciones no son prioridad.

De esta manera, la atención penal seguirá estando, hasta nuevo aviso, asentada en algunas bases tradicionales, por ejemplo, la reproducción de estereotipos sobre las mujeres y sus comportamientos. Nuestra sociedad, con sus castigos, no ha 
encontrado otra forma de hacer enmendar a las mujeres sus faltas, si no es sujetándolas a los valores tradicionales que se esperan de ellas. Es un tema que llama poderosamente la atención, porque, a pesar de que el acompañamiento psicológico y de trabajo social e incluso el del cuido de las mujeres a través de la policía penal es fundamental, la mirada hacia ellas sigue siendo estereotipada. En muchas ocasiones, he presenciado regaños hacia ellas, las llaman "manipuladoras" o las ignoran en sus peticiones.

No podría afirmar, en mi experiencia de investigadora, que hay un trato violento directo, pero sí se evidencia un lugar social en el cual son colocadas. Y eso no es sencillo, porque somos personas educadas para mirar la delincuencia con desprecio, por lo que no se puede asumir que el trabajo penitenciario anula, elimina, esta visión de mundo sobre las personas. La mirada institucional, al ser producto de la misma opresión, está cargada con todos los estigmas que se tienen sobre el delito y sobre la delincuencia. Incluso como antropóloga, el trabajo de desaprender todas las ideas que tenía sobre las mujeres en la cárcel implicó una gran tarea de cuestionamiento de los parámetros culturales con los cuales había crecido. Es innegable que el sistema penal construye relaciones de subordinación, a la vez que sostiene el poder de la relación sexo-género. No solo tiene que ver con el trato o el "maltrato" hacia ellas, sino con la consciencia de reconocer que el sistema penal refuerza la manera en cómo se ubica a las mujeres en la sociedad, lo que se dice y se espera de ellas.

Por otro lado, pensando en que el grueso de la población que entra a la cárcel es por el delito de tráfico de drogas, me pregunto si es el encierro el modo de enmendar el impacto económico de la desigualdad. ¿Existe un tratamiento para el delito de tráfico de drogas que logre una reinserción social? Si el tráfico de drogas es un delito sin sujeto, porque lo que se penaliza es el daño a la salud pública, ¿cómo se logra "tratar" a una persona que lo que tiene es un problema de opresión social? ¿Cómo se le enseña a alguien a "respetar" la salud pública si es un bien por lo demás abstracto?

Este enfoque en el trato penitenciario pasa por asumir que la reclusión de mujeres implica la reclusión de madres y, por lo tanto, la de algunos de sus hijos e hijas. Sin embargo, he notado una mirada naturalizada sobre lo que debe ser el sufrimiento penal y lo que las mujeres deberían hacer. De parte de algunas personas que ofrecen el cuido penal he escuchado: "Diay, si no quería sufrir por los hijos lo hubiera pensado antes". Delante de este estigma, podría afirmar que las mujeres sí lo pensaron muchas veces, antes de cometer un delito. Procuraron resolver, de mil formas, pero, al final, el tráfico les ofreció lo que el resto de la sociedad 
parece negarles en términos de creación de posibilidades económicas, entre otras. El tráfico sí es una oportunidad, no la mejor por su exposición a la cárcel, pero una salida económica, al fin y al cabo. Junto a ello, mientras que el cuidado de la prole y otras personas dependientes, tanto económica como emocionalmente, siga en manos de las mujeres, necesitamos pensar en recursos no privativos de libertad, para evitar que la mujer que comete un delito se desarraigue de su círculo primario.

Y es que el impacto de la cárcel es muy fuerte para quienes están adentro, pero hay una gran desestructuración en todos los que se quedan afuera. La marca más grande de la economía del tráfico está concentrada en la privación de libertad del último eslabón de la cadena. Cuando una mujer se encuentra pronta a salir de prisión con el cumplimiento de una media pena, o bien con el otorgamiento de un beneficio, se le pide una serie de requisitos, los cuales dudo que se puedan cumplir. Por ejemplo, cuando han estado vinculadas a la venta, en ocasiones se les solicita que no regresen a sus barrios y, además, que hagan una oferta laboral viable, la cual les asegure al sistema su decente "inserción”. Me pregunto: ¿cómo dejamos en las manos de personas desatendidas por el Estado que sean ellas mismas las que resuelvan en soledad aquello que este les negó estructuralmente (casa segura cuando salgan de la cárcel, una comunidad sin riesgos, un trabajo digno)?

La atención institucional penal tiene que poner su esmero más en la exclusión estructural y la desigualdad, no en el discurso de la pobreza material. Esta última se ha amalgamado a las personas, a las cuales se responsabiliza de su falta de recursos y "poca voluntad" para el trabajo, sin mirar las condiciones en las que ocurre esta exclusión. Las mujeres no comenten un delito por ser pobres, sino por estar excluidas y marginadas socialmente. Ellas no son pobres, están pobres. No son marginales, están marginadas. No son vulnerables, la sociedad las ha vulnerabilizado y, por lo tanto, el sistema penal no debe contribuir, con una mirada castrante que profundice la desigualdad estructural.

Un tema prioritario para el sistema penal, relacionado con la exclusión social de las mujeres, tiene que ver con las tareas productivas que ellas desarrollan en el centro, ya sean recreativas o vinculadas a una empresa. La gran mayoría de estas no tienen un enlace para su futura inserción laboral una vez que salgan de la cárcel. Esta se convierte en una queja de las féminas, pues dicen que en las cárceles para hombres se ofrece el trabajo en cuero, madera, artesanías comerciales que tienen el plus agregado de ser un producto de calidad fabricado por hombres en la cárcel. ¿Y las mujeres qué hacen? ¿Qué se les enseña? 
Los talleres productivos del centro penal se miran como un medio de pasar el tiempo, a la vez que quienes participan ganan algún dinero, una forma de sentirse menos oprimidas en el día tras día de las celdas. La prisión es un estigma y no se puede olvidar que ellas, los hombres también, que vienen de contextos privativos y marginales ya tienen una idea distorsionada de sí mismos, como privados de libertad. Esto implica que las mujeres no se puedan proyectar a sí mismas como sujetas productoras a través del trabajo digno, ese que les diera satisfacción, pues nunca tuvieron uno así antes de llegar a la cárcel, bien pagado y valorado y ahora, allí, es una idea que no se modifica. Las pocas reclusas que acceden al trabajo pagado en prisión lo hacen también en las mismas condiciones precarias que un trabajo mal pagado y explotador en la calle. Son conscientes de que la institución lo permite, lo avala en su negociación con las empresas privadas que llegan a la cárcel a ofrecer un convenio que tiene un beneficio unidireccional.

Estas mujeres arman cajas, bolsas de papel y por esto ganan, en unas horas, una suma que no cubre un salario mínimo, a pesar de tener jornadas de ocho horas. Habría que preguntarse si no ganan lo suficiente porque se cree que es parte de su castigo. En la cárcel he escuchado decir que, de todas maneras, las mujeres no tienen necesidad de gastar tanto dinero porque ahí cuentan con "techo" y "comida". Esta perspectiva lo que permite es afirmar que el Estado consiente el pago miserable de parte de la empresa privada, lo cual lo hace cómplice de reproducir aquello que quiere erradicar: el trabajo informal y explotador; a la vez que legitima una estructura de desigualdad salarial y de explotación laboral. ¿Por qué el Estado no hace convenios con sus otras instituciones? ¿Por qué no se mandan a fabricar más pupitres para las escuelas? ¿Por qué no se contrata a las mujeres, y hombres, en cooperativas que se encarguen de los comedores infantiles? Se puede hacer más, desde fabricar muebles, uniformes y hasta el trabajo en la construcción. Si bien algunas de estas acciones suceden, son aisladas y voluntarias de quien contrata, no masivas ni forman parte de la reinserción social que también sirva para la laboral.

Otro tema prioritario que deber ser asumido por la institución de privación de libertad es el control constante de la salud de las mujeres. Se debería contar con un diagnóstico de ingreso que permita construir un protocolo de atención total, pues el bienestar de esas mujeres en ese campo está en manos del Estado. Ni el papanicolaou ni la mamografía deberían ser voluntarios, sino obligatorios, preventivos y de acceso temprano. En alguna ocasión, también he escuchado a lo interno del centro penal: "Pero estas mujeres tampoco iban al Seguro cuando estaban afuera de la cárcel". La diferencia entre una persona institucionalizada y otra que no lo 
está radica en que sobre la primera el Estado corre con la responsabilidad de su bienestar, cuando hablamos de derechos humanos.

A este diagnóstico protocolario debe ir aparejada la detección de violencia. Si se piensa que hay estadísticas que hablan de la agresión concentrada en algunos espacios deprimidos socialmente y que estas mujeres podrían proceder de algunas de dichas zonas segregadas u olvidadas, nos encontramos en la cárcel con féminas que han vivido, en algún momento, una situación de abuso sexual o violencia física. Esto no sirve para explicar el delito, sino para procurar la prevención de la misma agresividad que puedan estar recibiendo del círculo familiar más cercano. Lo antes dicho no es porque una mujer violentada sea más propensa a llegar a la cárcel, más bien, porque es una forma en que el sistema penitenciario y de justicia puede contener, mediante redes institucionales, la reproducción de esa violencia a la que ellas se exponen.

En este mismo aspecto, el sistema penal debe atender la automedicación sedativa de las mujeres. Si bien hablar sobre el empleo de sustancias es un tema en sí mismo y se puede dejar para otra ocasión, es necesario revisar el consumo por medicación a través de sedantes facilitados por personal médico autorizado. Un estudio sobre el uso de sustancias ilícitas a lo interno de la cárcel para mujeres en el 2014 reveló que el consumo de medicamentos es alarmante (Murillo, García y Rodríguez, 2014). Este no solo se vincula a la afectación de la salud, sino a las redes económicas que también ingresan estas sustancias al centro penal; venderlas se convierte en parte de la economía ilícita interna, vinculada a la condición emocional dentro de la cárcel. Por otro lado, recetar tranquilizantes se puede estar convirtiendo en un síntoma de control social interno en los centros penales.

Finalmente, interesa referirse a la desproporcionalidad de las penas en el delito del tráfico de drogas. Algunas mujeres cumplen condenas de hasta 20 años por violación a la Ley 8204, que en algunas ocasiones se pueden superar por acumulación de procesos. Sin bien existe una modificación a la normativa que se empezó a aplicar en el 2013 con la Ley 9161 (Asamblea Legislativa, 2013), conocida como 77 Bis en su momento, contribuyó al descenso de la población penal, por introducir la proporcionalidad y especificidad de género en las penas por tráfico, a la vez que, a partir del 2018, se cuenta con la modificación al Código Penal en sus artículos 71 y 72, con los cuales se insta a tomar en cuenta las condiciones de vulnerabilidad de las mujeres para reducir las penas (Asamblea Legislativa, 2019).

Así mismo, hoy por hoy los números no son favorables. A finales del 2018, se llegó a un récord en la población penal, en la cárcel para mujeres Vilma Curling, 
con lo cual se superó históricamente su capacidad instalada en un $8 \%$ (Cascante, 2018). Esto podría estarnos enfrentando a una ola represiva sobre la privación de libertad, pues también existen modificaciones en los criterios para el otorgamiento de medidas alternativas a la prisión, con el que se dejan menos personas en libertad, bajo un discurso de apoyo a la seguridad. Si el Ministerio de Justicia está interesado en el ahorro económico al país, qué mejor que la implementación de otras disposiciones para implementar su estatus monetario como institución.

De las 684 mujeres recluidas en el Vilma Curling, al menos 366 están por tráfico de drogas. A partir de este hecho, si a esa población se aplican medidas alternativas a la prisión y si se toma en cuenta que una persona privada de libertad está valorada en por lo menos de 63 dólares diarios, tal vez se podría generar un ahorro, grosso modo, de ocho millones de dólares al año, que podrían ser invertidos en la creación de oportunidades sociales y educativas, en beneficio de estas mismas personas y sus familias.

Las mujeres que están en la cárcel no son diferentes a otras oprimidas socialmente en el país. Sus voces, sus necesidades y sus tristezas pertenecen al mismo orden de aquellas que día tras día tratan de idear maneras para pertenecer, social, económica y políticamente, a una nación que les ha negado oportunidades. Retomar su voz, con el propósito de contribuir a la equidad social, es una obligación.

\section{Referencias}

Asamblea Legislativa. (2001). Ley 8204. Reforma integral a la Ley sobre estupefacientes, sustancias psicotrópicas, drogas de uso no autorizado, actividades conexas, legitimación de capitales y financiamiento al terrorismo. San José: Diario Oficial La Gaceta.

Asamblea Legislativa. (2013). Ley 8204. Reforma integral a la Ley sobre estupefacientes, sustancias psicotrópicas, drogas de uso no autorizado, actividades conexas, legitimación de capitales y financiamiento del terrorismo, para introducir la proporcionalidad y especificidad de género, 9161. San José.

Asamblea Legislativa. (2019). Reforma integral Ley sobre estupefacientes, sustancias psicotrópicas, drogas de uso no autorizado, actividades conexas, legitimación de capitales y financiamiento al terrorismo. Recuperado de: http:// www.pgrweb.go.cr/scij/Busqueda/Normativa/Normas/nrm_texto_completo. aspx param $1=$ NRTC\&nValor $1=1 \&$ nValor $2=48392 \&$ nValor $3=0 \&$ strTipM $=\mathrm{TC}$ 
Cascante, L. (2018). Ministra de Justicia no acata medidas correctivas de jueces. Semanario Universidad.

Norbert, E. (1993). El proceso de civilización. Investigaciones sociogenéticas y psicogenéticas. México: Fondo de Cultura Económica.

Fernández, A. (1993). La mujer de la ilusión. Pactos y contratos entre hombres y mujeres. Buenos Aires: Paidós.

Instituto Costarricense sobre Drogas (ICD). (2009). Mujeres infractoras a la Ley de Psicotrópicos recluidas en el Centro de Atención Institucional El Buen Pastor. San José: Ministerio Justicia.

Juliano, D. (2004). Excluidas y Marginales. Madrid: Ediciones Cátedra.

Juliano, D. (2011). Presunción de inocencia. Riesgo, delito y pecado en femenino. Donostia: Gakoa Liburuak.

Murillo, B., García, C. y Rodríguez, A. (2014). Relación entre el delito y el consumo de droga en mujeres sentenciadas en el Centro Penal Buen Pastor. San José: Instituto Costarricense sobre Drogas (ICD).

Palma, C. (2018). Me puse a jugar de narco. Mujeres, tráfico de drogas y cárcel en Costa Rica. San José: Editorial de la Universidad de Costa Rica.

Pateman, C. (1988). The sexual contract. Stanford: Stanford University Press.

Programa de las Naciones Unidas para el Desarrollo Humano (PNUD). (2005). (In)seguridad ciudadana y desarrollo humano en Costa Rica. Venciendo el temor. Informe sobre desarrollo humano 2005. San José.

Sistema Integral de Atención Penitenciaria (SIAP). (2019). San José: Ministerio de Justicia.

Torres, A. (2008). Drogas, cárcel y género en Ecuador: La experiencia de mujeres "mulas”. Quito: ABYA-YALA, FLACSO. 
